\title{
O Uso de Uma Plataforma Virtual como Forma de Auxílio aos Discentes Durante o Distanciamento Social
}

\author{
Andressa Werner Krause Pereira ${ }^{1}$, Ana Amélia Traversi ${ }^{1}$, Gabriel Rocha Silveira ${ }^{1}$, \\ Raquel de Souza Zimmer ${ }^{1}$, Leomar Soares da Rosa Júnior ${ }^{1}$ \\ ${ }^{1}$ Centro de Desenvolvimento Tecnológico \\ Universidade Federal de Pelotas (UFPel) - Pelotas - RS - Brasil \\ \{awkpereira, aatraversi, grsilveira, rdszimmer, \\ leomarjr\}@inf.ufpel.edu.br
}

\begin{abstract}
Due to the need for social distancing caused by the COVID-19 pandemic, education suffered a rupture in traditional patterns of student and teacher meetings in classrooms, which consequently led to a fraternal distancing between individuals. Such concerns were taken into account by Grupo PET Computação which, as a solution, conceived a platform capable of sustaining the link created by remote classroom teaching. To achieve this goal, the Discord Application was chosen, which with its various tools and friendly interface has become a virtual space for study and interaction for students and professors.
\end{abstract}

Resumo. Devido a necessidade do afastamento social causado pela pandemia da COVID-19, o ensino sofreu uma forte ruptura com padrões tradicionais de reuniões de alunos e professores em salas de aula, o que por consequência acarretou o afastamento fraterno entre os indivíduos. Tais preocupações foram abraçadas pelo grupo PET Computação que, como solução, idealizou uma plataforma capaz de sustentar o vínculo criado pelo ensino presencial de forma remota. Para realizar este objetivo foi escolhido o Aplicativo Discord, que com suas diversas ferramentas e interface amigável tornou-se um espaço virtual de estudo e interação para alunos e professores.

\section{Introdução}

Com origem em Wuhan, China, o vírus coronavírus se alastrou rapidamente pelo mundo, fazendo com que escolas e universidades cancelassem suas atividades presenciais em concordância com a quarentena [Agência Brasil 2020] [UFPEL(a) 2020]. A UFPel, diante da impossibilidade de continuar suas aulas em caráter presencial, organizou-se para continuar suas atividades remotamente [UFPEL(b) 2020].

Após a organização das atividades, no entanto, era preciso dirigir-se a outro ponto importante do aprendizado - a motivação dos alunos. O semestre alternativo começou em 22 de junho de 2020, 3 meses após a interrupção do primeiro semestre e 6 meses após o começo das férias [UFPEL(c) 2020] - um período de tempo significativo sem aulas e sem contato com a maioria dos colegas.

Com o objetivo de facilitar as interações universitárias durante a pandemia, o grupo de Programa de Educação Tutorial - Computação da Universidade Federal de Pelotas decidiu utilizar-se de um ambiente interativo e de fácil uso, o aplicativo de comunicação conhecido como Discord. 
O Discord provou-se a ferramenta ideal para lidar com estes problemas. Ele é um aplicativo gratuito e de fácil acesso, disponível no navegador, no aplicativo e no celular. Ele também é uma mistura interessante entre os aplicativos antigos de conversa, como o Skype e o MSN, e com os programas mais populares, como o Google Meet. Sua dinâmica de servidores é excelente para encontros dinâmicos entre grupos diversos.

Não só no caso deste artigo, o Discord tornou-se comument utilizado no ensino durante o período. Contudo uma diferenciação entre o caso aqui redigido e alguns mais comuns apresentados no meio científico, é a obrigatoriedade do uso da plataforma, como Monica Vladoiu em seu artigo "Learning during COVID-19 Pandemic: Online Education Community, based on Discord", que expôs o uso do Discord como principal meio de transmissão de conhecimento, execução de trabalhos e etc., o objetivo aqui foi o uso complementar, mais próximo àquele apresentado por Joyce, Randall; Sahawneh, Faris; and Dixon, Brandon em "Utilizing Discord to Create a Learning Space for Cybersecurity Courses".

\section{Metodologia}

Com a necessidade de afastamento físico, tornou-se parte da proposta do PET Computação UFPel manter a proximidade intelectual. Para isso, foi de vital importância o uso de uma ferramenta de comunicação online multifuncional, que permitisse interações entre os membros e mantivesse um ambiente limpo para comunicação e organização dos projetos do grupo. Nesses moldes, o Discord foi escolhido para execução de reuniões e discussões, pois, segundo documentos da própria plataforma, "O Discord é um aplicativo gratuito de bate-papo por voz, vídeo e texto usado por dezenas de milhões de pessoas com mais de 13 anos para conversar e sair com seus amigos e comunidades" [Discord Safety 2021].

A boa adaptação de usuários novos e antigos ao método de trabalho fomentou a ideia de ampliação do uso da plataforma. E com a proposta de um semestre remoto pela UFPel, tornou-se prioridade para o grupo não permitir que o distanciamento ocasionasse quebras na comunicação entre alunos e professores dos cursos de Computação. Assim, foi idealizado o "Servidor da Computação", com intuito inicial de fornecer um espaço extraoficial dinâmico e organizado de comunicação e estudo.

O início da criação do servidor - espaço virtual destinado a conversas por texto e áudio, no qual é possível a organização hierárquica dos participantes - se deu pela escolha do nome "Computação UFPEL" e inserção de uma imagem representativa (Figura 1).

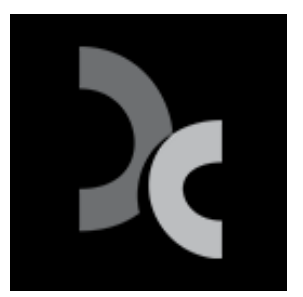

Figura 1. Logo dos cursos de Computação da Universidade Federal de Pelotas. 
Em seguida, para suprir a necessidade geral de interação entre os alunos, foi criado um canal - canais são explicados pela seguinte ideia "Os servidores Discord são organizados em canais de texto e voz, dedicados a tópicos específicos e podem ter regras diferentes" [Discord Safety 2021], no qual os participantes podem conversar da forma que desejarem. Da mesma forma, para organizar os espaços de estudos, cada uma das disciplinas ofertadas recebeu um canal de texto e voz, os quais são restringidos somente a professores, monitores e alunos inscritos na cadeira e tem objetivo específico de ser um canal de comunicação para tópicos pertinentes. Durante o período de engajamento analisado, um canal de texto foi suficiente para suprir as necessidades, o mesmo ocorreu para os canais de voz.
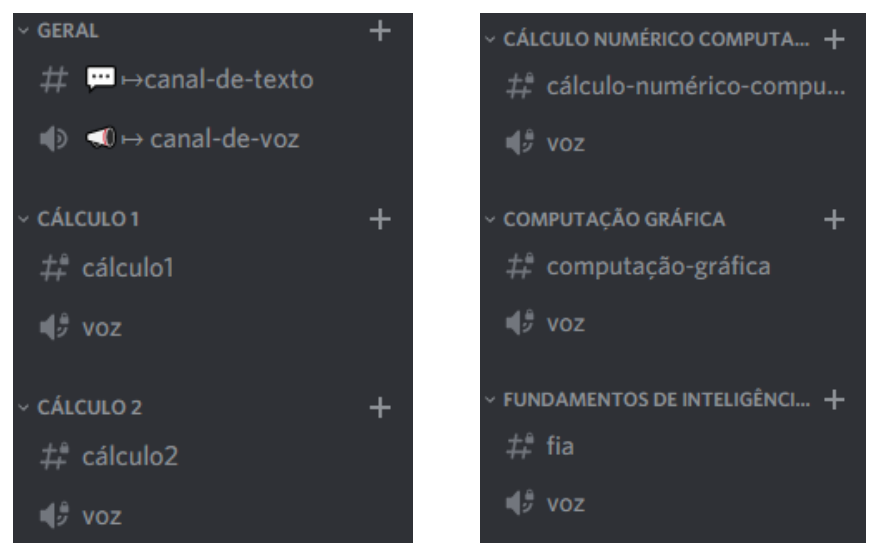

Figura 2. Exemplos de páginas de texto e voz categorizadas de acordo com as cadeiras oferecidas.

O sistema de conversa por texto é bastante atrativo para os alunos e professores, pois além de possibilitar ambas conversações curtas, centradas em mensagens assíncronas e conversações síncronas, com possível participação de diversas pessoas; inclusive, conta com sistema de threads em que conversas paralelas podem ser discutidas em tópicos facilmente; o ambiente também torna-se agradável devido a possibilidade do uso de emojis, gifs e figurinhas que "têm como objetivo principal expressar uma ideia ou sentimento por meio de mensagem de forma simples e rápida, que não através do uso de palavras, e sim elementos gráficos” [Medeiros 2020]. 


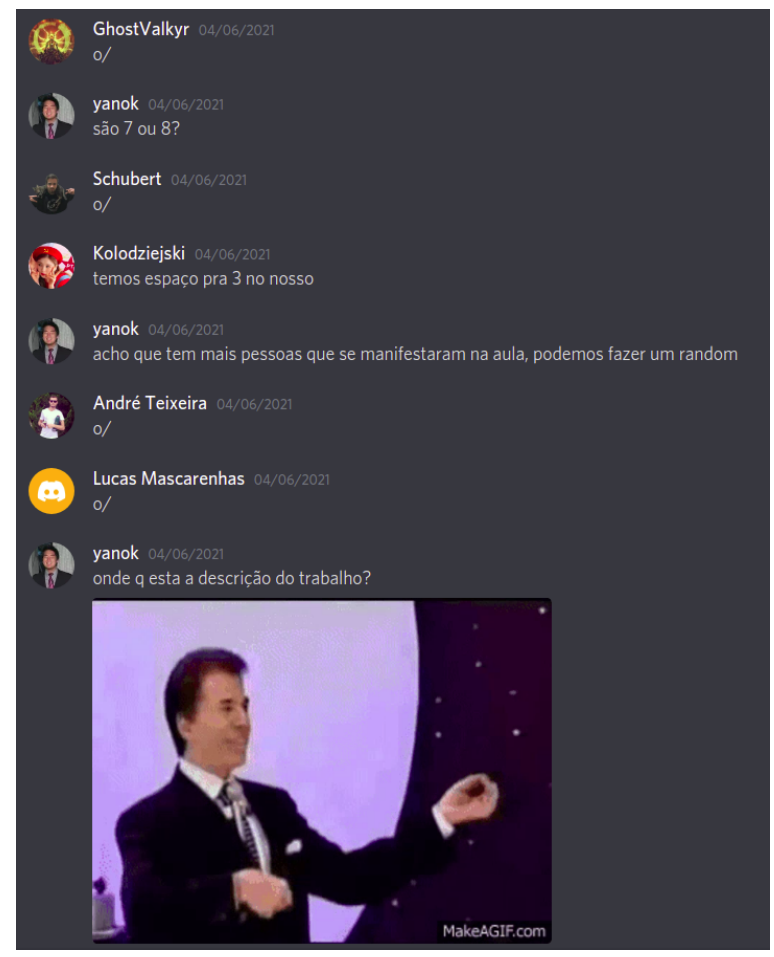

Figura 3. Uma conversa informal entre alunos de uma disciplina, com uso de emoticons (o/, que representa uma pessoa com o braço levantado) e um gif. 0 emoticon foi usado para representar uma ação, aquela de levantar a mão, enquanto o gif foi usado para descontrair a pergunta.

Por sua vez, as conversas por voz tornaram-se uma forma de imitar as conversas face-a-face, pois permitem interação simultânea dos usuários. Conversas que exigem exposições longas e são complicadas de serem expressas por texto, tornam-se viáveis por meio de canais de voz e, além disso, a plataforma oferece serviços de vídeo e de compartilhamento de tela (Figura 4), os quais permitem que um participante mostre aos demais o que está fazendo em seu dispositivo, possibilitando, assim, exploração de conteúdos e detalhadas explicações, pois diversas mídias podem ser incorporadas.

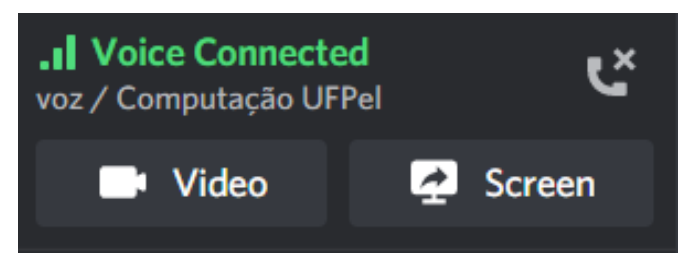

Figura 4. Os participantes podem identificar que estão conectados a um canal de voz através do menu mostrado na figura, o qual dá acesso às ferramentas de vídeo e compartilhamento de tela, além de informar em qual canal e servidor o participante está e como está a qualidade de sua conexão.

Para organização do servidor foi implementado um sistema de rótulos, nos quais os participantes são capazes de inscrever-se nas matérias desejadas. A ideia não só identificou os membros, mas também foi um recurso para ajudar os participantes a manterem uma visualização mais limpa do servidor, uma vez que tem acesso somente às 
páginas nas quais estão inscritos. O processo de rotulação foi implementado de forma que cada indivíduo escolhesse quais páginas gostaria de acessar através de cliques em botões designados. Essa automatização se deu através da implementação de um bot, que é um programa programado para realizar tarefas predeterminadas repetidamente.

Foram implementados, também, páginas com as regras do servidor, para inscrições e para comunicados. A página de regras tem por finalidade informar àqueles recém chegados sobre os princípios básicos do servidor; inscrições possibilitam a visualização das cadeiras disponíveis e a efetivação da participação das categorias desejadas; e comunicados faz uma ponte entre os administradores do servidor e os demais integrantes, nela são feitos anúncios e avisos.

\section{Resultados e Discussão}

A plataforma, até o momento da escrita deste artigo, foi utilizada por um ano e seis meses como servidor de interação da computação. Durante este período, foi alcançado o marco de 581 participantes. Notou-se que este servidor foi procurado também por pessoas com deficiência visual, que interagem normalmente com outros membros, o que o torna inclusivo e acessível para esta comunidade, já que a plataforma conta com funções de acessibilidade, como o recurso TTS que faz a leitura de textos.

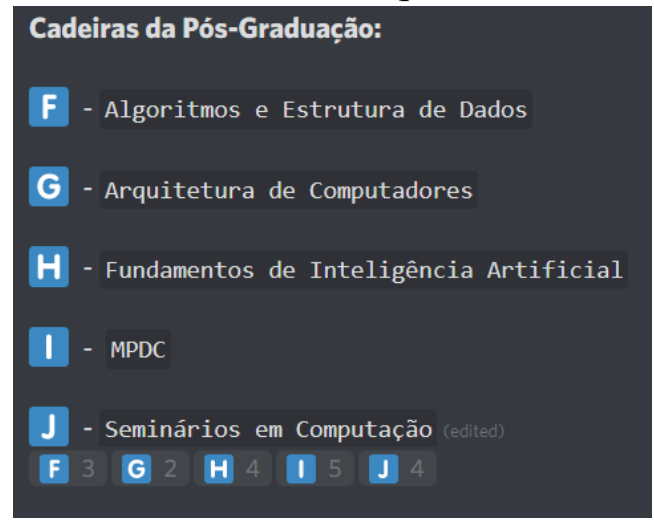

Figura 5. Exemplos de cadeiras para inscrição dos participantes.

Segundo pesquisa realizada com os usuários da plataforma(Figura 6), na qual obtemos 23 respostas, percebeu-se que além da plataforma ser utilizada para estudos, também auxiliou em manter o vínculo dos alunos com a universidade no momento em que o distanciamento social está vigente. Tal conexão ocorreu através de avisos de lives relacionadas ao curso e a universidade, salas de bate papo, prática de jogos online, juntamente com alunos e professores, trazendo uma relação mais próxima entre os mesmos, dentre outros. 


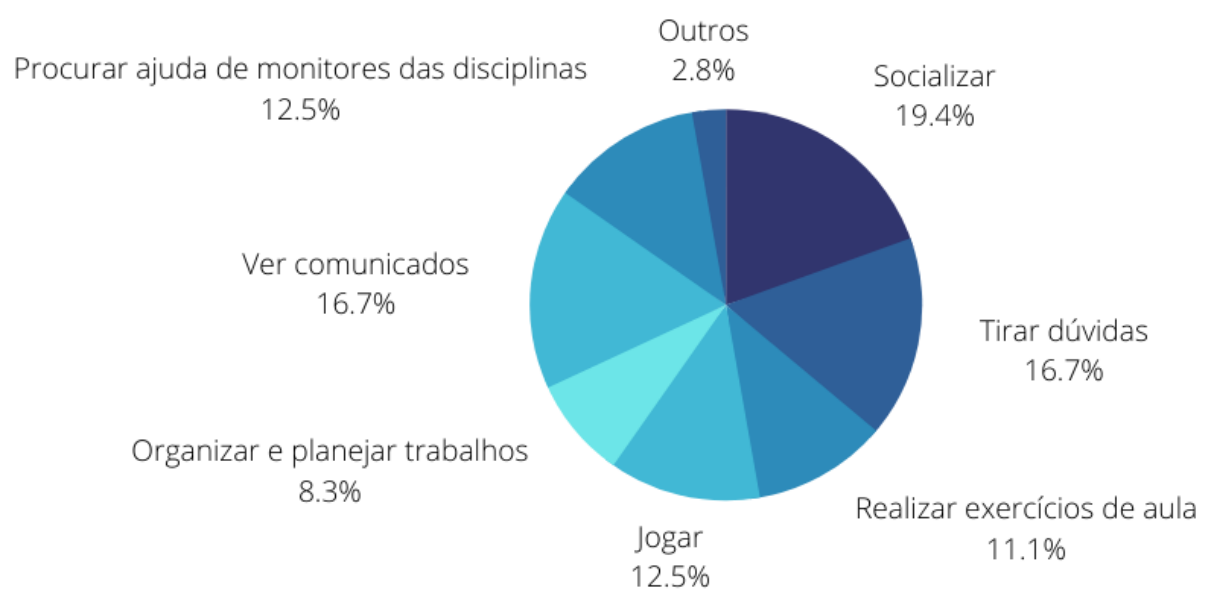

Figura 6. Utilização do Discord da Computação.

A criação da sala para jogos ocorreu em meio a pedidos de grande parte dos usuários, então foi feita uma consideração cuidadosa dos administradores, onde acabou decidindo-se pela criação de um espaço inteiramente focado em lazer coletivo, mas que não comprometeria aqueles que desejassem focar-se inteiramente nos estudos. Todos os participantes receberam a opção de inscrever-se em um conjunto de páginas categorizadas como "Jogos online". Como resultado, tivemos várias interações entre alunos e professores, além de terem sido criados eventos de jogos cooperativos, que desenvolvem a interação social, trabalho em equipe e raciocínio lógico.

Visto que a ideia inicial deste servidor era amparar os estudantes durante as aulas online, considera-se que o objetivo foi alcançado com sucesso, como mostrado pela Figura 7. A maioria das pessoas pesquisadas sentiu-se auxiliada de alguma maneira pela plataforma, sendo para tirar dúvidas, procurar monitores, desenvolver projetos ou estudar junto de seus colegas fora do horário de aula.

Para que o formulário fosse mais assertivo, foram inseridas apenas questões alternativas, com exceção da última, que coleta sugestões de melhoria para o servidor. Por isto, não foram coletadas informações sobre o porquê das respostas "Não" para as Figuras 7 e 8.

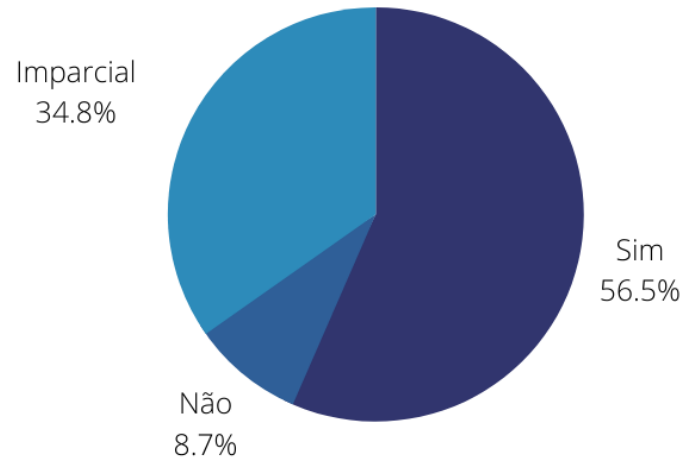

Figura 7. O servidor ajudou nas aulas EAD? 
Por conta das várias possibilidades que o servidor do Discord possui e da unificação de vários recursos utilizados em aula, tais como canais de voz e chat, compartilhamento de tela, envio de arquivos, dentre outros, o número de adeptos cresceu rapidamente. Professores e alunos passaram a utilizar a ferramenta como via de comunicação e interação, considerando a ferramenta importante para o espaço acadêmico durante as aulas remotas, como mostra a Figura 8.

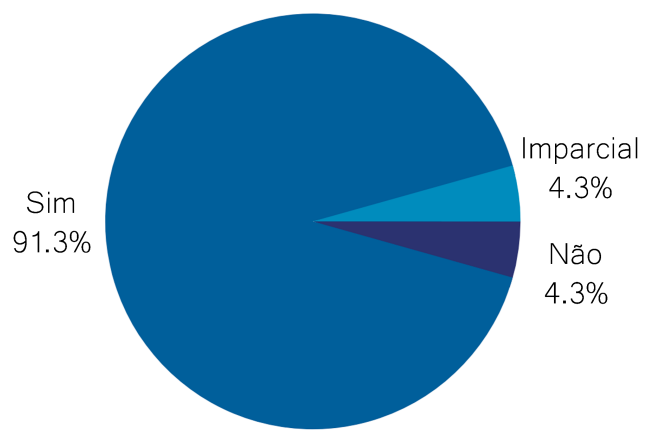

Figura 8. Considera o Discord uma ferramenta importante no espaço acadêmico durante o período EAD?

\section{Conclusões}

O objetivo deste artigo foi relatar a implementação do Servidor da Computação no Discord, durante o primeiro semestre de Calendário Acadêmico Remoto na UFPel em virtude da pandemia da COVID-19. Através do questionário aplicado e das percepções que foram obtidas no decorrer do projeto, nota-se que a comunicação entre os alunos foi facilitada neste período de distanciamento social. O servidor foi amplamente utilizado pela comunidade acadêmica de computação da UFPel, disseminando a informação e fomentando a interação entre discentes e docentes, mostrando que o projeto desenvolvido pelo Grupo PET Computação teve êxito em sua execução. Outro indicativo de sucesso do projeto foi o fato de diversos alunos terem solicitado para que o projeto tenha continuidade mesmo quando a Universidade retornar suas atividades presenciais.

Ao longo do período de utilização, que atualmente soma cerca de 18 meses, receberam-se sugestões de melhorias, oriundas da comunidade, como, por exemplo, canais exclusivos de monitoria e cargos para monitores, os quais foram aderidos, facilitando a comunicação dos envolvidos.

Para os próximos semestres o Grupo PET Computação pretende dar continuidade neste projeto, independentemente do retorno às aulas presenciais ou não, contando com alterações das categorias a cada início de período letivo, a fim de ajustá-las com as disciplinas ofertadas no semestre. Espera-se que a iniciativa continue aproximando, mesmo que virtualmente, todos os envolvidos.

\section{Referências}

Agência Brasil. Organização Mundial da Saúde declara pandemia de coronavírus. Agência Brasil, Brasília, 11 mar. 2020. Notícias. Disponível em: 
$<$ https://agenciabrasil.ebc.com.br/geral/noticia/2020-03/organizacao-mundial-da-sau de-declara-pandemia-de-coronavirus $>$ Acessado em 20 set. 2021.

M. Vladoiu and Z. Constantinescu, "Learning During COVID-19 Pandemic: Online Education Community, Based on Discord," 2020 19th RoEduNet Conference: Networking in Education and Research (RoEduNet), 2020, pp. 1-6, doi: 10.1109/RoEduNet51892.2020.9324863.

Joyce, Randall; Sahawneh, Faris; and Dixon, Brandon, "Utilizing Discord to Create a Learning Space for Cybersecurity Courses" (2021). Pedagogicon Poster Gallery. 8. $<$ https://encompass.eku.edu/pedagogicon_postergallery/8>

Discord Safety. O que é o Discord?. Discord. Disponível em: $<$ https://discord.com/safety/360044149331-O-que-e-o-Discord> Acessado em 19 set. 2021.

MEDEIROS, Alex Sandro Peixoto. Multimodalidade Textual: um avanço sociolinguístico no processo comunicativo digital com o uso de emojis, gifs e figurinhas. Brasil Escola. Disponível em: $<$ https://monografias.brasilescola.uol.com.br/educacao/multimodalidade-textual-umavanco-sociolinguistico-no-processo-comunicativo-digital-com-o-uso-de-emojis-gifs -e-figurinhas.htm\#indice_9> Acessado em 19 set. 2021.

UFPEL(a). CRA reforça que calendário acadêmico está suspenso. Portal UFPel, Pelotas, 2 abr. 2020. Notícias. Disponível em: $<$ https://ccs2.ufpel.edu.br/wp/2020/04/02/cra-reforca-que-calendario-academico-esta -suspenso/> Acessado em 20 set. 2021.

UFPEL(b). Cocepe aprova calendário alternativo. Portal UFPel, Pelotas, 22 maio. 2020. Notícias. Disponível em: $<$ https://ccs2.ufpel.edu.br/wp/2020/05/22/cocepe-aprova-calendario-alternativo-ativi dades-iniciam-em-22-6/> Acessado em 20 set. 2021.

UFPEL(c). Reitor da UFPel realiza vídeo ao vivo para falar sobre o coronavírus. Portal UFPel, Pelotas, 16 mar. 2020. Notícias. Disponível em: $<$ https://ccs2.ufpel.edu.br/wp/2020/03/16/reitor-da-ufpel-realiza-video-ao-vivo-para-f alar-sobre-coronavirus/> Acessado em 20 set. 2021. 